\title{
Anesthetic Approach in Placental Invasion Anomalies
}

\author{
Aslan Bilge ${ }^{1 *}$, Aydın Feray², Gedikli Ahmet ${ }^{1}$ and Uygur Dilek ${ }^{3}$ \\ ${ }^{1}$ Anesthesia Clinic Department, Zekai Tahir Burak Training and Research Hospital, University of Health Sciences, Ankara, Turkey \\ ${ }^{2}$ General Surgery, Clinic of Zekai Tahir Burak Training and Research Hospital, Turkey \\ ${ }^{3}$ Perinatologist, Close Care Risky Pregnancy Department, Tahir Burak Training and Research Hospital, University of Health Sciences, Ankara, Turkey
}

"Corresponding author: Bilge Aslan, Anesthesia Clinic Department, Zekai Tahir Burak Training and Research Hospital, University of Health Sciences, Ankara, Turkey, Tel: +90 312 3065184; E-mail: drbilgeaslan@hotmail.com

Received: 17 Jan, 2019 | Accepted: 03 May, 2019 | Published: 09 May, 2019

Citation: Bilge A, Feray A, Ahmet G, Dilek U (2019) Anesthetic Approach in Placental Invasion Anomalies. Gynecol Women's Health Res 1(1): dx.doi. org/10.16966/2689-3096.111

Copyright: ( 2019 Bilge A, et al. This is an open-access article distributed under the terms of the Creative Commons Attribution License, which permits unrestricted use, distribution, and reproduction in any medium, provided the original author and source are credited.

Keywords: Placental invasions; Anesthesia management

\section{Introduction and Purpose}

Peripartum hemorrhage takes place within the significant reasons for maternal morbidity and mortality. Abnormal placentation is one of the significant reasons for peripartum hemorrhage. The basic forms of abnormal placentation are placenta previa and abnormal invasive placenta [1]. The optimum time for planned delivery for a patient with placenta accreta is around 34-35 weeks following corticosteroid injection. The successful management of placenta accreta includes a multidisciplinary care team approach relying heavily on the prenatal diagnosis of this entity and preparing for surgical management. The aim of this study was to investigate of the cases Caesarean Section $(\mathrm{C} / \mathrm{S})$ with the diagnosis of placenta previa and abnormal invasive placenta in terms of anesthesia management, transfusion requirement and problems predicted. The anesthesiologist should act according to the condition of the pregnant and plan the case by communicating with other departments. In such cases, regional anesthesia is sufficient in terms of quality and duration of analgesia and is preferred in our clinic.

\section{Materials and Methods}

After the approval of the ethics committee, the records of the patients who underwent cesarean section with the diagnosis of placenta previa/accreta in our hospital between 2008-2018 were reviewed. Demographic data and pregnancy characteristics of the patients (Table 1), type of operation, duration of operation, surgical intervention in cesarean section, perioperative haemodynamic data, ephedrine, vasopressor, crystalloid or colloid consumption. Also, perioperative Erythrocyte Suspension (ES), Fresh Frozen Plasma (FFP), Thrombocyte Suspension (TS) consumption, Preoperative and Postoperative Hemoglobin (Hb), Thrombocyte, Partial Thromboplastin Time (PT), Activated Partial Thromboplastin Time (aPTT), International Normalized Ratio (INR), Alanine Transaminase (ALT) and Aspartate Aminotransferase (AST), Creatine (Cr) values, and the lowest measured hemoglobin value were recorded. Different treatment modalities are applied for peripartum hemorrahage in abnormal placental invasion. Uterine curretage, tamponade devices, peripartum hysterectomies, uterine artery ligation, hypogastric artery ligation, and hypogastric artery ballooning are the examples for the treatment. In our study, we have percentages of treatment methods and percentages of reoperation.

\section{Results}

A total of 1200 cases were found $2.5 \%(n=30)$ of the cases were placenta accreta, 3.5\% $(n=42)$ were placenta percreata; $64 \%(n=768)$ were placenta previa totalis and $30 \%(n=360)$ were placenta previa marginalist. $48 \%(n=576)$ of the cases were regional, $50 \%(n=600)$ were general, $2 \%(n=24)$ were applied regional and general together. The mean operation time was $60 \pm 24$ minutes (Table 2). Caesarean was applied to only $80 \%(n=960)$ of the cases, additionally to caesarean intrauterine balloon application for $15 \%(n=180)$ of cases, hysterectomy for $5 \%(n=60)$. Repeat operation was needed for $5 \%$ $(n=60)$ of the patients. Preoperative mean Hb value was $12.00( \pm 0.8)$ g.dl ${ }^{-1}$, platelet value was $210000( \pm 8020) \mu \mathrm{l}^{-1}$, INR value was $1.4( \pm$ $0.2)$; postoperative mean $\mathrm{Hb}$ value was $10.8( \pm 0.5)$ g.dl-1 ${ }^{-1}$, thrombocyte value was $150560( \pm 4250) \mu \mathrm{l}^{-1}$, INR value was $1.2( \pm 0.1)$. Erythrocyte suspension for $40 \%(n=480)$ of patients, TDP for $30 \%(n=360)$, thrombocyte suspension for $1 \%(n=12)$ and fibrinogen was used for $6 \%(n=72)$. In this study, Student-t test was used in statistical evaluation, where numerical variables were not equally distributed between groups; Mann-Whitney $U$ test was used in different distributions. There were significant changes in $\mathrm{Hb}$, platelet and aPTT values $(\mathrm{p}<0.05)$. There were significant decreases in these three values ( $\mathrm{Hb}$, platelet, aPTT) postoperatively. The intraoperative blood loss and transfusion requirements of patients' placenta accreta and its variants undergoing cesarean delivery (Tables 3-5).

\section{Discussion and Conclusion}

Placenta previa is an obstetric complication that placenta covers the internal cervical OS or placed near the OS, which has an incidence 
Table 1: Demographic data and pregnancy characteristics of patients. Data mean given as \pm standard deviation given (minimum-maximum), n (\%).

\begin{tabular}{|l|c|}
\hline Age (years) & $30 \pm 12(18-42)$ \\
\hline Height $(\mathrm{cm})$ & $160 \pm 8(150-174)$ \\
\hline Weight $(\mathrm{kg})$ & $80 \pm 14(43-134)$ \\
\hline Pregnancy week $(\mathrm{wk})$ & $36 \pm 4(24-40)$ \\
\hline Number of pregnancy $(\mathrm{n})$ & $3 \pm 2(0-5)$ \\
\hline Number of births $(\mathrm{n})$ & $0.8 \pm 1.2(0-10)$ \\
\hline Number of previous cesarean $(\mathrm{n})$ & $2 \pm 1(0-3)$ \\
\hline $\begin{array}{l}\text { Number of patients underwent uterine surgery } \\
(\mathrm{n}, \%)\end{array}$ & $120(\% 10)$ \\
\hline
\end{tabular}

Table 2: Diagnosis and operation time of patients. Data mean given as \pm standard deviation given (minimum-maximum), $\mathrm{n}(\%)$.

\begin{tabular}{|l|c|}
\hline Placenta accreta $(n, \%)$ & $30(2.5 \%)$ \\
\hline Placenta percreata $(n, \%)$ & $42(3.5 \%)$ \\
\hline Placenta previa totalis (n,\%) & $768(64 \%)$ \\
\hline Placenta previa marginalis (n,\%) & $360(30 \%)$ \\
\hline $\begin{array}{l}\text { Type of anesthesia ( } n \text {,\%) Regional, Time of } \\
\text { discharge }\end{array}$ & $576(48 \%), 6( \pm 2)$ day \\
\hline $\begin{array}{l}\text { Type of anesthesia ( }, \%) \text { General, Time of } \\
\text { discharge }\end{array}$ & $600(50 \%), 10( \pm 3)$ \\
\hline $\begin{array}{l}\text { Type of anesthesia (n,\%) Regional+General, } \\
\text { Time of discharge }\end{array}$ & $24(2 \%), 9( \pm 3)$ day \\
\hline Duration of operation (min) & $60 \pm 24(30-230)$ \\
\hline Surgical period (min) & $56 \pm 24(22-245)$ \\
\hline Induction-delivery time (min) & $4 \pm 2.1(2-12)$ \\
\hline
\end{tabular}

Table 3: Type of surgery applied to patients. Data were given as $n$ (\%).

\begin{tabular}{|l|c|}
\hline Only cesarean $(n, \%)$ & $960(80 \%)$ \\
\hline Intrauterine balloon application $(n, \%)$ & $180(15 \%)$ \\
\hline Hysterectomy $(n, \%)$ & $60(5 \%)$ \\
\hline Bladder repair $(n, \%)$ & $1(0.4 \%)$ \\
\hline Patients applied second operation $(n, \%)$ & $60(5 \%)$ \\
\hline
\end{tabular}

of $0.3-0.6 \%$, is also a risk factor for abnormal invasive placentation [2]. Placenta accreta is one of the important reasons for abnormal invasive placentation postpartum hemorrhage that covers the increta and percreta and it is related to prominent maternal mortality. Surgical morbidity can depend on the degree of the placental invasion. The incidence of the abnormal invasive placentation has been increased from $0.08 \%$ to $0.3 \%$ in last 10 years depending on the caesarean ratio increase [3].

The risk of abnormal invasive placentation is higher when the caesarean with placenta previa is occurred. Regional anesthesia for cesarean delivery in women with placenta previa is safe [4]. Delivery should take place at an institution with adequate blood banking facilities. The incidence of placenta accreta is rising, primarily because of the rise in cesarean delivery rates. This condition can be associated with massive blood loss at delivery. Prenatal diagnosis by imaging, followed by planning of peripartum management by a multidisciplinary team, may help reduce morbidity and mortality. Women known to
Table 4: Perioperative hemodynamics, ephedrine usage, liquid, blood, blood product consumptions and the lowest measured hemoglobin values. Data mean given as \pm standard deviation given (minimummaximum), $n(\%)$.

\begin{tabular}{|l|c|}
\hline Pressure systolic $(\mathrm{mmHg})$ & $125( \pm 12)(60-120)$ \\
\hline Pressure diastolic $(\mathrm{mmHg})$ & $60( \pm 15)(40-80)$ \\
\hline Heart $\uparrow$ (beats/min) & $110( \pm 12)(70-160)$ \\
\hline Heart $\downarrow$ (beats/min) & $80( \pm 16)(45-125)$ \\
\hline Patients developing hypotension $(\mathrm{n}, \%)$ & $720(60 \%)$ \\
\hline Patients used ephedrine (n,\%) & $360(30 \%)$ \\
\hline Ephedrine (mg) & $20( \pm 10)(0-40)$ \\
\hline Patients used fibrinogen (n,\%) & $12(4.6 \%)$ \\
\hline Fibrinogen (g) & $0.06( \pm 0.04)$ \\
\hline Patients used erythrocyte suspension $(\mathrm{n}, \%)$ & $480(40 \%)$ \\
\hline Erythrocyte suspension (U) & $4( \pm 2)$ \\
\hline Patients used fresh frozen plasma (n,\%) & $360(30 \%)$ \\
\hline Fresh frozen plasma (U) & $4( \pm 1)$ \\
\hline Patients used thrombocyte $(\mathrm{n}, \%)$ & $12(1 \%)$ \\
\hline Thrombocyte (U) & $2( \pm 0.5)$ \\
\hline Crystalloid (ml) & $2000( \pm 112)$ \\
\hline Colloid (ml) & $800( \pm 150)$ \\
\hline Lowest hermoglobin (g.dl $\left.{ }^{-1}\right)$ & $9( \pm 3.5)(6-12.5)$ \\
\hline
\end{tabular}

Table 5: Laboratory results before and after surgery. Data mean given as \pm standard deviation given (minimum-maximum).

\begin{tabular}{|l|c|c|}
\hline & Before the operation & After the operation \\
\hline Hemoglobin $\left(\mathrm{g} . \mathrm{dl}^{-1}\right)^{*}$ & $12.00( \pm 0.8)(7-13.5)$ & $10.8( \pm 0.5)(7-13.5)$ \\
\hline Platelet $\left(\mu \mathrm{I}^{-1}\right)^{* *}$ & $\begin{array}{c}210000( \pm 8020) \\
(80000-350000)\end{array}$ & $\begin{array}{c}150560( \pm 4250) \\
(60000-250000)\end{array}$ \\
\hline PT (second) & $12.5( \pm 0.5)(10-24)$ & $12.9( \pm 0.1)(10-19.7)$ \\
\hline aPTT (second)*** & $26.1( \pm 0.1)(16-45)$ & $31.6( \pm 0.6)(21-99)$ \\
\hline INR (U) & $1.4( \pm 0.2)(0.9-1.27)$ & $1.2( \pm 0.1)(0.8-1.9)$ \\
\hline AST & $17( \pm 1.1)(10-250)$ & $20( \pm 2)(10-388)$ \\
\hline ALT & $18( \pm 0.7)(10-180)$ & $25( \pm 5)(10-212)$ \\
\hline Cr $\left(m g . d l^{-1}\right)$ & $0.8( \pm 0.4)$ & $1.2( \pm 0.2)$ \\
\hline
\end{tabular}

$*, * *, * * *=\mathrm{Hb}$, Platelet and a PTT preoperative and postoperative values $\mathrm{p}<0.05$

have placenta accreta should be delivered by cesarean, and no attempt should be made to separate the placenta at the time of delivery. The majority of women with significant degrees of placenta accreta will require a hysterectomy [5].

Complete placenta previa is associated with an increased risk of hemorrhage compared with incomplete placenta previa. Therefore the distinction between classifications should be factored into anesthetic management protocols [6].

The choice of anesthetic technique in patients with abnormal placentation is controversial. Even in patients who are taken to cesarean with the indication of placenta previa, general anesthesia is more common due to the possibility of bleeding, but there are publications showing that regional anesthesia is safe in these patients. Bleeding amount of the abnormal invasive placentation cases is changing between the median values of 2000-6000 $\mathrm{ml}$ which are quite high numbers. 
In our study, there was no awakening in patients receiving regional anesthesia. The duration of stay in the ICU of the pregnant women was shorter. Therefore, regional anesthesia decreases mortality. It shortens the time of discharge of pregnant women.

Regardless of the anesthetic technique used, it is recommended for abnormal placentation diagnosed patients that are going to be taken to caesarean, to be performed at least two large vascular access, arterial cannulation should be performed and central venous cannulation should be considered if necessary. Two to five units of erythrocyte suspension must be available. It should be kept in mind that vasoactive drugs such as phenylephrine, ephedrine, and dopamine may be needed [7]. It should be kept in mind that major obstetric hemorrhages may be encountered in cases with abnormal placentation and the necessity of dynamic anesthesia management. Regional anesthesia for cesarean delivery in women with placenta previa is safe. The risk of abnormal invasive placentation is higher when the caesarean with placenta previa is occurred. This condition can be associated with massive blood loss at delivery. If the operation is prolonged or a complication develops, we have the option of limiting regional anesthesia and passing to general anesthesia. In such cases, regional anesthesia is sufficient and preferred for the quality and duration of analgesia. If the pre-operative preparation is complete for the abnormal invasive placentation, the anesthesiologist can reduce mortality and morbidity.

\section{References}

1. Kuczkowski KM (2011) A Review of the current anesthetic concerns and concepts for cesarean hysterectomy. Curr Opin Obstet Gynecol 23: 401-407.

2. Silver RM (2015) Abnormal Placentation: Placenta Previa, Vasa Previa, and Placenta Accreta. Obstet Gynecol 126: 654-668.

3. Brookfield KF, Goodnough LT, Lyell DJ, Butwick AJ (2014) Perioperative and transfusion outcomes in women undergoing cesarean hysterectomy for abnormal placentation. Transfusion 54: 1530-1536.

4. Kocaoglu N, Gunusen I, Karaman S, Ergenoglu A, Firat V (2012) Management of anesthesia for cesarean section in parturients with placenta previa with/without placenta accreta: A retrospective study. Ginekol Pol 83: 99-103.

5. Oyelese Y, Smulian JC (2006) Placenta previa, Placenta Accreta, and Vasa Previa. Obstet Gynecol 107: 927-941.

6. Orbach-Zinger S, Weiniger CF, Aviram A, Balla A, Fein S, et al. (2018) Anesthesia management of complete versus incomplete placenta previa: a retrospective cohort study. J Matern Fetal Neonatal Med 31: 1171-1176.

7. Kuczkowski KM (2006) Anesthesia for the repeat cesarean section in the parturient with abnormal placentation: what does an obstetrician need to know? Arch Gynecol Obstet 273: 319-321. 